\title{
Latent stimulus control develops in extinction after very brief feature-negative, but not feature-positive, discrimination training in the runway
}

\author{
STEVEN J. HAGGBLOOM \\ Arkansas State University, State University, Arkansas
}

\begin{abstract}
Two groups of 10 rats each were briefly trained on a feature-discrimination learning problem in the runway. In Group FP, the feature was present on positive (S+) trials and absent on negative (S-) trials. The opposite arrangement held for Group FN. After 12 learning trials, 7 with S+ and 5 with S-, both groups received 10 extinction trials in a single session, 5 with $\mathrm{S}+$ and 5 with S-. Neither group showed evidence of differential responding to $S+$ or $S-$ in acquisition. In extinction, Group FN ran faster on S+ trials than on S- trials, an effect called latent discrimination learning. There was no evidence of latent discrimination learning in Group FP. The results suggest that impaired FP performance in the runway, relative to FN performance, reflects retarded learning on the FP discrimination, rather than a performance deficit.
\end{abstract}

Under some discrimination learning conditions, differential stimulus control by the positive $(\mathrm{S}+)$ and negative (S-) discriminative stimuli is not evident during discrimination training itself, yet it develops during a subsequent phase of extinction (Capaldi, 1979; Capaldi, Berg, \& Morris, 1975; Hearst, 1987, 1988). The emergence of differential response tendencies with respect to $\mathbf{S}+$ and $\mathbf{S}-$ during an extinction test is called latent discrimination learning. Some of the reasons why discrimination learning may be latent have been discussed by Capaldi and Hearst and are not of immediate concern here. Rather, the present experiment capitalized on the fact that the procedures developed by Capaldi et al. (1975) provide a means of detecting differential control by $\mathrm{S}+$ and $\mathrm{S}-$ after very brief runway discrimination training.

The purpose of the present experiment was to assess the early development of differential stimulus control in a feature-discrimination learning problem in the runway. In this task, the discriminative cue is the presence versus absence of a distinctive stimulus (feature). In a go/no-go discrimination task in the runway, discriminative responding develops faster in the feature-negative (FN) condition, in which the feature is a component of $S-$, than in the feature-positive (FP) condition, in which the feature is a component of S+ (Haggbloom, 1983; Haggbloom \& Sheppard, 1986). The faster rate of discrimination learning in the FN condition is called a feature-negative effect (FNE).

In situations other than the runway-as, for example, those involving keypecking by pigeons in an operant

This experiment was supported by a grant from the Arkansas State University Faculty Research Committee. Correspondence may be addressed to Steven J. Haggbloom, Department of Psychology, Arkansas State University, P.O. Box 2127, State University, AR 72467.
chamber-FP learning is invariably superior to FN learning, and, indeed, FN learning may completely fail to develop (see Hearst, 1978; Jenkins \& Sainsbury, 1969). However, Hearst $(1987,1988)$ recently reported that discriminative responding that is absent or impaired during training on an FN problem will develop or improve during extinction. This latent FN discrimination learning reflects the acquisition of differential control by $\mathrm{S}+$ and $\mathrm{S}-$ that was not evident in behavior during discrimination training.

In the runway, the FP discrimination is eventually learned, and, given adequate training, performance in the FP condition approaches that in the FN condition (Haggbloom, 1983, Experiment 1). The superior FN performance shows up early in training, after as few as 24 trials (Haggbloom, 1983, Experiment 2). The purpose of the present experiment was to look for latent discrimination learning following very abbreviated FP and FN training in the runway. Evidence for latent FP learning following limited training would indicate that impaired performance on FP discriminations in the runway is due to deficient control of FP responding rather than to retarded FP learning.

The procedures used here were modeled after those used by Capaldi et al. (1975), who reported latent development of differential stimulus control by $\mathrm{S}+$ (a black runway) and S- (a white runway) in extinction after only 12 discrimination learning trials. The important procedural feature of their experiment was the presentation of all daily $\mathrm{S}+$ trials prior to daily $\mathrm{S}-$ trials. This trial sequence minimizes the conflicting stimulus control that can be exercised by internal reward-produced cues, and it thereby facilitates the detection of differential control by S+ and S- (Capaldi et al., 1975; Haggbloom, 1978; Haggbloom \& Tillman, 1980). 
In the present experiment, two groups of rats, labeled FP and FN, were trained in the runway on feature-positive and feature-negative discriminations, respectively. Both groups received a total of 12 discrimination learning trials, 7 with $S+$ and 5 with $S-$, over 4 days of training, followed on Day 5 by a single session of 10 extinction trials, 5 with each discriminandum.

\section{METHOD}

\section{Subjects}

The subjects were 20 male rats, bred in the laboratory from Holtzman stock, 90-120 days old at the start of discrimination training. Ten rats were randomly assigned to each group.

\begin{abstract}
Apparatus
The apparatus consisted of a single straight alleyway that was $235 \mathrm{~cm}$ long, $10 \mathrm{~cm}$ wide, and $15 \mathrm{~cm}$ high. The alleyway, made of wood, had a hinged hardware-cloth top. The initial and final $25 \mathrm{~cm}$ of the runway constituted start- and goalboxes, respectively. The start- and goalboxes were separated from the rest of the runway by manually operated guillotine doors. Three clocks $(.01 \mathrm{sec})$ recorded start, run, and goal times over respective distances of 20,150 , and $30 \mathrm{~cm}$ from the startbox door. Opening the startbox door activated the first clock, and photoelectric circuitry controlled its offset and the operation of the remaining two clocks. The goalbox contained an unpainted goal cup constructed by drilling a 6-cm hole in a small block of wood. The goal cup was positioned against the end wall of the goalbox. The feature was a tactile cue that consisted of $1 / 2$ in. hardware cloth laid over the floor of the startbox and runway between the startbox and goalbox doors.
\end{abstract}

\section{Procedure}

The rats were housed individually and placed on a $12-\mathrm{g}$ per day diet of lab chow 2 weeks prior to the start of discrimination training. On each of the 3 days immediately preceding training, the rats were handled in squads of 2 for $2 \mathrm{~min}$ and later fed six $.045-\mathrm{g}$ food pellets in their home cages.

There were 12 discrimination training trials, 7 with $\mathrm{S}+$ and 5 with $S-$. The trial sequence over Days $1-4$ consisted of,,+-++-++- , and ++-- . On $S+$ trials, the rats received six $.045-\mathrm{g}$ food pellets, and on $S-$ trials, they were confined to the unbaited goalbox for $20 \mathrm{sec}$. For Group FP, the presence of the tactile cue constituted S+ and its absence was S-. The opposite arrangement held for Group FN. On Day 5 , each rat received a rewarded $S+$ trial followed by 10 extinction trials, 5 each with $S+$ and $S-$. The sequence of extinction trials was +-++-+--+- for half the rats in each group and the reverse of this sequence for the remaining rats.

The rats were run in squads of 4 , containing 2 rats from each group. The intertrial interval was approximately $4 \mathrm{~min}$. A maximum time of $60 \mathrm{sec}$ was allowed in each alley section. If $60 \mathrm{sec}$ were exceeded in any alley section, the additional time was added to the time for the next section. If a rat did not enter the goalbox within $180 \mathrm{sec}$, the trial was terminated by placing the rat in the goalbox, where it received the scheduled trial outcome.

\section{RESULTS}

All analyses were performed on running times converted to speeds in centimeters per second. Differences were most pronounced in the goal section of the runway, so only goal speeds are reported here.

There was no difference between mean running speeds on $\mathrm{S}+$ and $\mathrm{S}-$ trials for either group on any day of discrimination training. For Group FP, the differences between mean running speeds on $\mathrm{S}+$ and $\mathrm{S}$ - trials on Days $1-4$ were $-5.57,-0.64,-3.45$, and $2.21 \mathrm{~cm} / \mathrm{sec}$, re- spectively. The comparable values for Group FN were $-5.83,-4.08,0.28$, and $-0.54 \mathrm{~cm} / \mathrm{sec}$. Separate analyses of variance with groups (2) as a between-subjects variable and discriminanda (2) as a within-subjects variable were applied to mean $S+$ and $S$ - speeds on each day of discrimination training. None of the analyses showed significant main effects or groups $X$ discriminanda interactions (all $F s<1$ ).

Figure 1 shows the mean running speed on the five extinction trials with $S+$ and the five extinction trials with $\mathrm{S}-$ for both groups. As can be seen, Group FP ran at very nearly the same speed on S+ and S- trials. Group FN, on the other hand, ran as fast as Group FP on S+ trials, but it ran slower on $\mathrm{S}-$ trials than on $\mathrm{S}+$ trials. A 2 (group) $\times 2$ (discriminanda) $\times 5$ (trial) analysis of variance applied to the data shown in Figure 1 yielded a reliable group $\times$ discriminanda interaction $[F(1,18)=4.47, p<$ .05]. Simple effects of discriminanda for each group showed that Group FN ran reliably faster in S+ than in $S-[F(1,18)=4.90, p<.05]$, but that $S+$ and $S-$ running speeds in Group FP did not differ $(F<1)$.

\section{DISCUSSION}

In the present experiment, differential responding to $S+$ and $S-$ that had not developed during a very brief discrimination training phase emerged during an extinction test in Group FN, which was trained on a feature-negative discrimination, but this did not occur in Group FP, which was trained on a feature-positive discrimination. The failure of latent discrimination learning to occur in Group FP suggests that the slower acquisition of FP discriminations relative to $\mathrm{FN}$ discriminations in the runway (Haggbloom, 1983; Haggbloom \& Sheppard, 1986) reflects impaired learning in the FP case, rather than a deficiency in the early control of FP performance.

The present results stand in contrast to those obtained in the operant chamber. In that preparation, FP performance is invariably superior to FN performance, a feature-positive effect (FPE), and the FPE may be a consequence of deficient control of discriminative responding in the FN case rather than impaired learning (Hearst, 1987). The runway preparation, on the other hand, uniquely and regularly yields superior

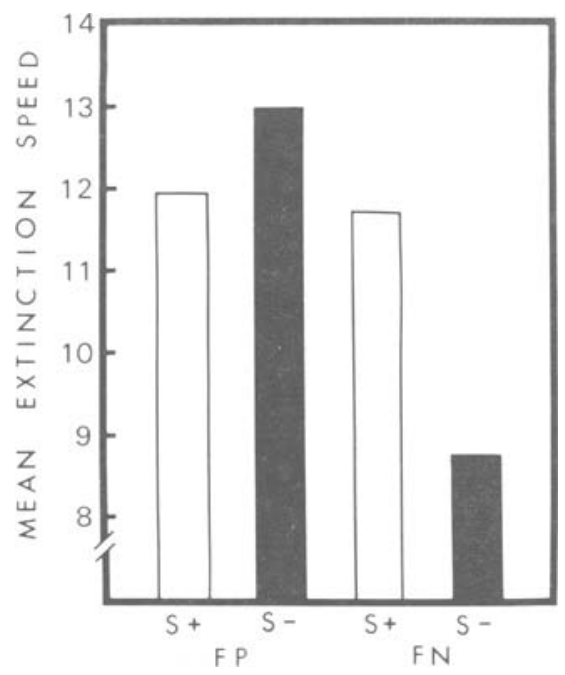

Figure 1. Overall mean running speed for Group FP and Group FN on extinction trials with $\mathrm{S}+$ and $\mathrm{S}-$. 
performance on the FN discrimination problem, an outcome that the present results suggest is due to faster learning in the FN case.

\section{REFERENCES}

CAPALDI, E. J. (1979). Latent discrimination learning under a regular schedule of partial reinforcement. Animal Learning \& Behavior, 7, 63-68.

CaPAldi, E. J., Berg, R. F., \& Morris, M. D. (1975). Stimulus control of responding in the early trials of differential conditioning. Learning \& Motivation, 6, 217-229.

HAGGBLOOM, S. J. (1978). Intertrial interval effects on internal stimulus control of behavior in brightness differential conditioning. Learning \& Motivation, 9, 347-358.

HAGGBLOOM, S. J. (1983). Feature-negative effect in rats' discrimination learning in the runway. Animal Learning \& Behavior, 11, 367-372.

HAGGBLOOM, S. J., \& SHEPPARD, F. K. (1986). Feature-negative effect in serial learning. Bulletin of the Psychonomic Society, 24, 217-218.
Haggbloom, S. J., \& Tillman, D. J. (1980). Sequential effects on discrimination reversal. Learning \& Motivation, 11, 318-338.

HeArST, E. (1978). Stimulus relationships and feature selection in learning and behavior. In S. Hulse, H. Fowler, \& W. K. Honig (Eds.), Cognitive processes in animal behavior (pp. 51-88). Hillsdale, $\mathrm{NJ}$ : Erlbaum.

HEARST, E. (1987). Extinction reveals stimulus control: Latent learning of feature-negative discriminations in pigeons. Journal of Experimental Psychology: Animal Behavior Processes, 13, 52-64.

HeArst, E. (1988). The feature-positive effect in pigeons: Conditionality, overall predictiveness, and type of feature. Bulletin of the Psychonomic Society, 26, 73-76.

Jenkins, H. M., \& Sainsbury, R. S. (1969). The development of stimulus control through differential reinforcement. In $\mathbf{N}$. J. Mackintosh \& W. K. Honig (Eds.), Fundamental issues in associative learning (pp. 123-161). Halifax, NS, Canada: Dalhousie University Press.

(Manuscript received June 9, 1989.)

\section{ERRATUM}

Heller, M. A., \& Kennedy, J. M. Perspective taking, pictures, and the blind (Bulletin of the Psychonomic Society, 1989, 27, 500 [Abstract No. 148]). In the program of the 30th Annual Meeting of the Psychonomic Society and in the reprint of the program included in the November 1989 issue of The Bulletin, the surname of the first author, Morton A. Heller, was misspelled as Meller. The incorrect spelling occurs also on page 534 in the Author Index and on page 537 in the Address Index. 